\title{
The scientific work of Vladimir Maz'ya
}

\section{Alberto Cialdea ${ }^{1}$}

Received: 12 October 2020 / Revised: 22 October 2020 / Accepted: 23 October 2020 /

Published online: 27 July 2021

(c) The Author(s) 2020

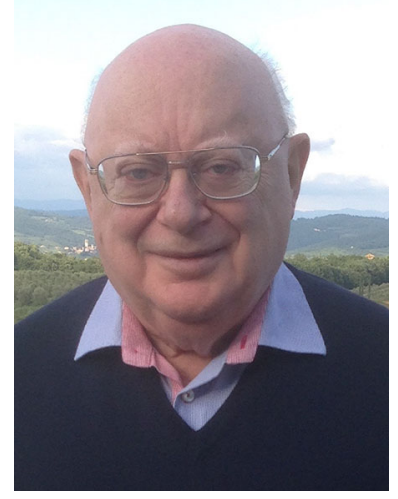

\section{Introduction}

Vladimir Maz'ya is an outstanding mathematician whose work had a profound impact on the modern analysis. In more than 60 years of activity, he wrote over 500 papers and 40 monographs. Figure 1 gives an impressive glance of his books.

The great depth of the results he has obtained, his fundamental new ideas, and his skilled technique characterize his papers.

Among his most important results are the discovery of the equivalence between Sobolev and isoperimetric/isocapacitary inequalities (which we shall describe in Sect. 2), his counterexamples related to Hilbert's 19th and 20th problems (see Sect. 3), his solution, together with Yuri Burago, of a problem in harmonic potential theory posed by Riesz and Nagy, his solution with Mikhail Shubin of a problem in the

\footnotetext{
Alberto Cialdea

cialdea@email.it

1 Department of Mathematics, Computer Sciences and Economics, University of Basilicata, V.le dell'Ateneo Lucano, 10, 85100 Potenza, Italy
} 


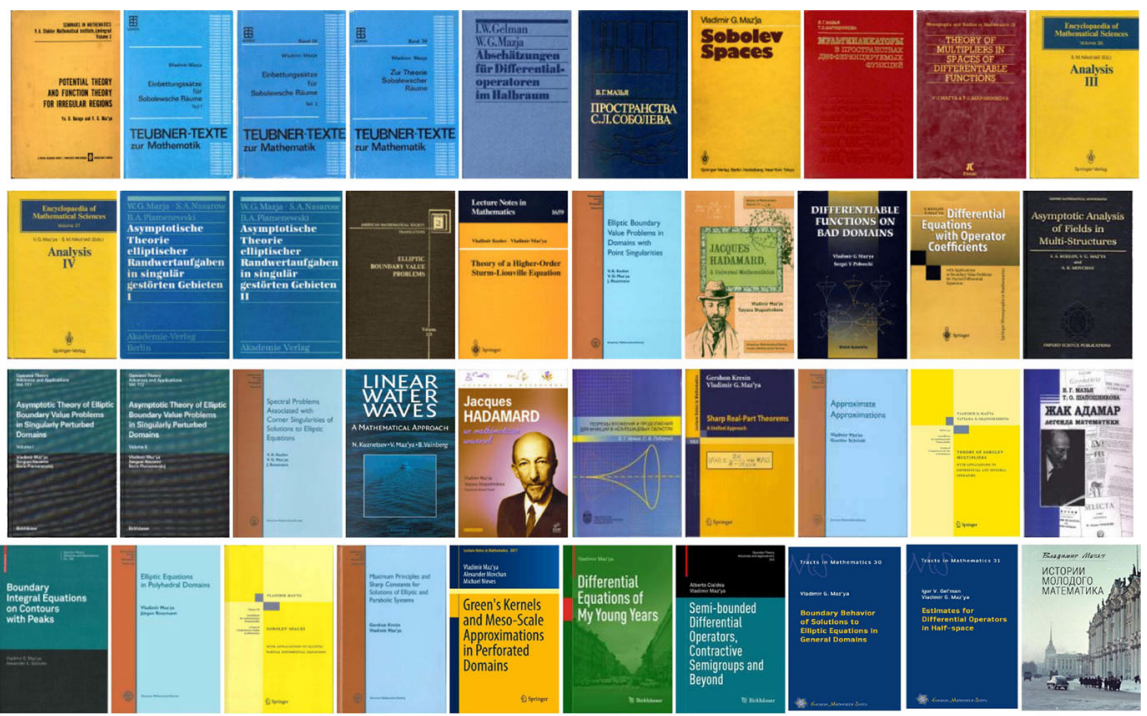

Fig. 1 Maz'ya’s books

spectral theory of the Schrödinger operator formulated by Gelfand, his solution with Victor Havin of a problem related to harmonic approximation posed by Mergelyan. He solved also Vladimir Arnol'd's problem for the oblique derivative boundary value problem and Fritz John's problem on the oscillations of a fluid in the presence of an immersed body. He gave also seminal contributions to the development of the theory of capacities, nonlinear potential theory, the asymptotic and qualitative theories of arbitrary order elliptic equations, the theory of ill-posed problems, the theory of boundary value problems in domains with piecewise smooth boundary. He introduced the concept of Approximate Approximation, which provides a completely new approach to fast numerical procedures.

He received several Honours and Awards:

- 1962, Prize of the Leningrad Mathematical Society, Russia.

- 1990, Doctor honoris causa of the University of Rostock, Germany.

- 1999, Humboldt Research Prize.

- 2001, Corresponding Fellow of the Royal Society of Edinburgh.

- 2002, Member of the Royal Swedish Academy of Sciences.

- 2003, Verdaguer Prize of the French Academy of Sciences.

- 2004, The Celsius Gold Medal of the Royal Society of Sciences at Uppsala.

- 2009, Senior Whitehead Prize of the London Mathematical Society.

- 2012, Elected Fellow of the American Mathematical Society.

- 2013, Foreign Member of the Georgian National Academy of Sciences.

His reputation is attested by several Conferences which have been organized in his honour:

- Sobolev spaces and potential theory. Conference in honour of Vladimir Maz'ya, Kyoto, Japan, 1993. 
- Functional Analysis, Partial Differential Equations and Applications. Conference in honour of Vladimir Maz’ya, Rostock, Germany, 1998.

- Analysis, PDEs and Applications. Conference on the occasion of the 70th birthday of Vladimir Maz’ya, Rome, Italy, 2008.

- Nordic-Russian Symposium in honour of Vladimir Maz'ya on the occasion of his 70th birthday, Stockholm, Sweden, 2008.

- Analysis of Partial Differential Equations, Symposium in honour of Vladimir Maz'ya on the occasion of his 75th Birthday, Liverpool, UK, 2013.

- Sobolev Spaces and Partial Differential Equations, on the occasion of the 80th birthday of Vladimir Maz'ya, Accademia dei Lincei, Rome, Italy, 2018.

- Harmonic Analysis and PDE, International Conference in honor of Vladimir Maz’ya, Holon, Israel, 2019.

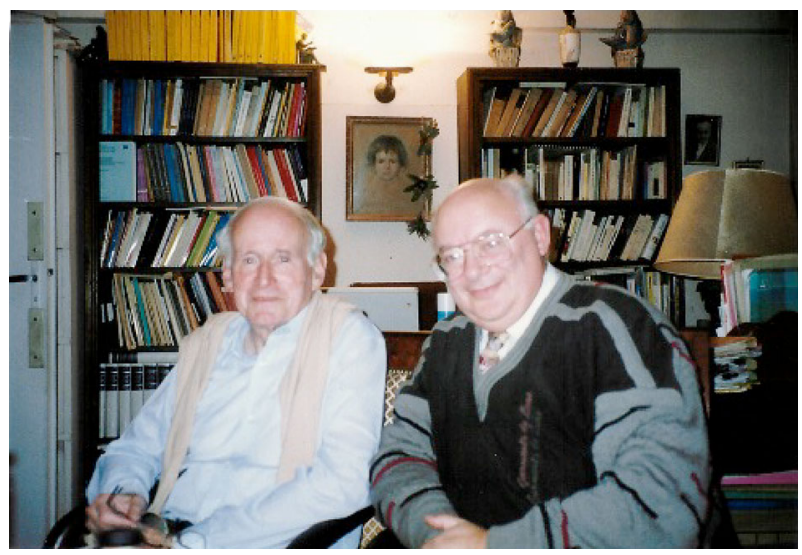

Laurent Schwartz and Vladimir Maz’ya (Paris, 1992)

Several books and papers have been dedicated to him:

- Two volumes of “The Maz’ya Anniversary Collection”, edited by Rossmann, J., Takač, P., Wildenhain, Birkhäuser, 1999.

- Mathematical Aspects of Boundary Element Methods, dedicated to Vladimir Maz'ya on the occasion of his 60th birthday, edited by M. Bonnet, A.M. Sändig and W. Wendland, Chapman \& Hall/CRC Research Notes in Mathematics, London, 1999.

- Perspectives in Partial Differential Equations, Harmonic Analysis and Applications: A Volume in Honor of Vladimir G. Maz'ya's 70th Birthday, edited by D. Mitrea and M. Mitrea, Proc. of Symposia in pure mathematics, Vol. 79, Amer. Math. Soc., Providence (R.I.), 2008.

Analysis, Partial Differential Equations and Applications. The Vladimir Maz'ya Anniversary Volume, edited by A. Cialdea, F. Lanzara, P.E. Ricci, Operator Theory, Advances and Applications, Vol. 193, Birkhäuser, Berlin, 2009.

- D. Eidus et al, Mathematical work of Vladimir Maz'ya (on the occasion of his 60th birthday), Funct. Differ. Equ. 4 (1997), no. 1-2, pp. 3-11.

- M.S.Agranovich et al, Vladimir G. Maz'ya, On the occasion of his 65th birthday, Russian Journal of Mathematical Physics, Vol. 10, No. 3, 2003, pp. 239-244. 
- M. S. Agranovich et al, Vladimir Gilelevich Maz'ya (on his 70th birthday), Russian Math. Surveys 63:1(2008), 189-196.

- M.V. Anolik et al, Vladimir Gilelevich Maz'ya (On the Occasion of his 70th Anniversary), Vestnik Sankt-Peterburgskogo Universiteta. Seriya 1. Matematika, Mekhanika, Astronomiya, no. 4 (2008), 3-6.

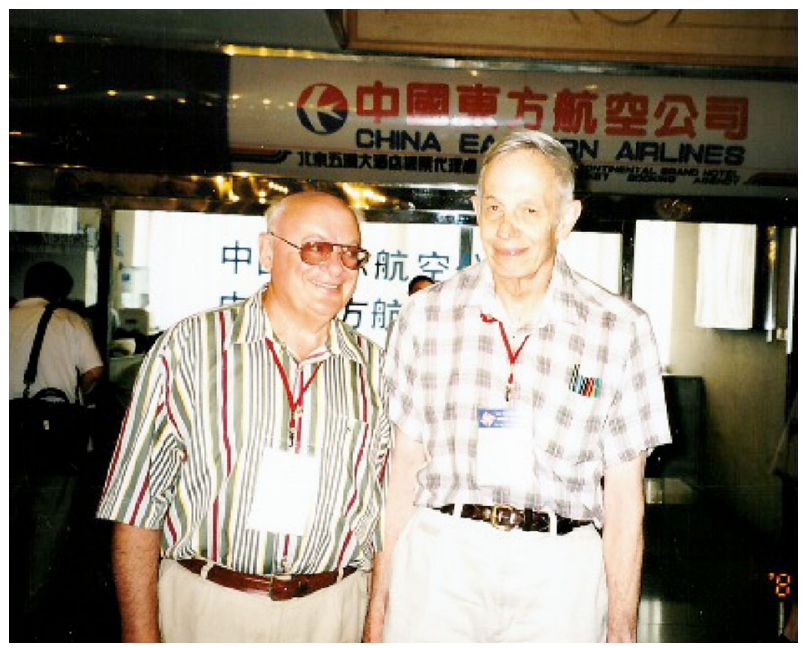

Vladimir Maz'ya and John Nash (Beijing, 2002)

One of the characteristic of Maz'ya scientific activity is the great variety of topics. This is only a tentative list of the subjects of his research:

1. Equivalence of isoperimetric and integral inequalities

2. Theory of capacities and nonlinear potentials

3. Counterexamples related to the 19th and 20th Hilbert problems

4. Boundary behaviour of solutions to elliptic equations in general domains

5. Non-elliptic singular integral and pseudodifferential operators

6. Degenerating oblique derivative problem

7. Estimates for general differential operators

8. Boundary integral equations

9. Linear theory of surface waves

10. The Cauchy problem for the Laplace equation

11. Theory of multipliers in spaces of differentiable functions

12. Characteristic Cauchy problem for hyperbolic equations

13. Boundary value problems in domains with piecewise smooth boundaries

14. Asymptotic theory of differential and difference equations with operator coefficients

15. Maximum modulus principle for elliptic and parabolic systems, contractivity of semigroups

16. Iterative procedures for solving ill-posed boundary value problems

17. Asymptotic theory of singularly perturbed boundary value problems

18. "Approximate approximations" and their applications 
19. Wiener test for higher-order elliptic equations

20. Spectral theory of the Schrödinger operator

21. Navier-Stokes equations

22. History of Mathematics

23. Mesoscale asymptotic expansion

24. Criteria for the accretivity and form boundedness of second order elliptic equations 25. Eigenfunctions of the Fourier transform

26. Sobolev spaces in unrestricted domains

Clearly, it is impossible to give a complete description of the scientific activity of Vladimir Maz'ya in one paper. I had to make some choices, also considering that certain topics will be discussed in other contributions by other participants of the meeting dedicated to his 80th anniversary.

Vladimir Maz'ya has a reputation of being the solver of problems which are generally considered as unsolvable. This is why Fichera once compared him with Santa Rita, the 14th century Italian nun who is the Patron Saint of Impossible Causes.

About twenty years ago Vladimir Maz'ya and Sergej Nikol'skij met at ISAAC Conference in Berlin (2001). I remember how Nikol'skij told to Maz'ya that he could not sleep because of a problem he could not solve. Maz'ya asked what problem was. Nikol'skij's answer was: I will not tell you, otherwise you will solve it !

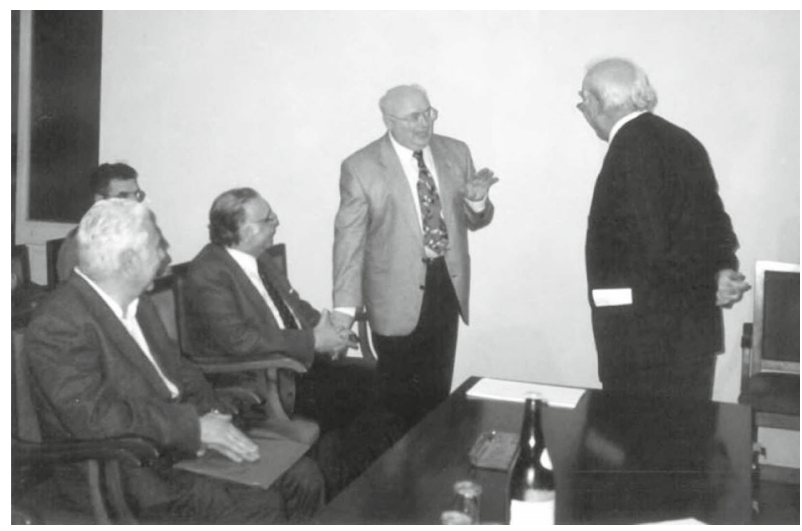

From left to right: Ennio De Giorgi, Gaetano Fichera, Vladimir Maz' ya and Giorgio Salvini (President of Accademia dei Lincei)

\section{Equivalence of isoperimetric/isocapacitary and integral inequalities}

Sobolev embedding theorems are well known. One of them asserts that if $1<p<n$, the following inequality holds

$$
\|u\|_{L^{p^{*}}(\Omega)} \leq C\left(\|\nabla u\|_{L^{p}(\Omega)}+\|u\|_{L^{p}(\Omega)}\right)
$$

for any $u$ in the Sobolev space $W^{1, p}(\Omega)$. Here $\Omega$ is a domain in $\mathbb{R}^{n}$ and $p^{*}=n p /(n-$ $p$ ). This result was proved by Sobolev and later extended to the case $p=1$ by 
Gagliardo and Nirenberg (1958). In these papers the domain $\Omega$ satisfies the so-called cone property. This means that each point of the domain is the vertex of a spherical cone with fixed height and angle which is situated inside the domain.

The inequality (1) does not hold on an arbitrary domain. For example, it is easy to see that if $\Omega=\left\{(x, y) \in \mathbb{R}^{2} \mid 0<x<1,0<y<x^{4}\right\}$, the function $u(x, y)=1 / x$ belongs to $W^{1, \frac{3}{2}}(\Omega)$, but not to $L^{6}(\Omega)$.

On the other hand there exist domains showing that cone property is not necessary for the validity of inequality (1).

At a very young age, when he was still a fourth year undergraduate student, Vladimir Maz'ya discovered the equivalence between Sobolev embeddings like (1) and isoperimetric and isocapacitary inequalities. The classical isoperimetric inequality states that for any planar domain $\Omega$ with a rectifiable boundary of a fixed lenght $L$ we have

$$
4 \pi A \leq L^{2}
$$

where $A$ is the area of $\Omega$. The equality holds if and only if $\Omega$ is a disk. The $n$-dimensional generalization of (2) is

$$
\left(\operatorname{mes}_{n} g\right)^{\frac{n-1}{n}} \leq C_{n} \mathcal{H}_{n-1}(\partial g)
$$

where $g$ is a domain in $\mathbb{R}^{n}$ with smooth boundary $\partial g$ and compact closure, and $\mathcal{H}_{n-1}$ is the $(n-1)$-dimensional area. The constant $C_{n}=n^{-1} v_{n}^{-1 / n}$ is such that (3) becomes equality for any ball (here $v_{n}$ denotes the volume of the unit ball). Maz'ya discovered that Sobolev inequality

$$
\|u\|_{L^{n /(n-1)}\left(\mathbb{R}^{n}\right)} \leq C_{n}\|\nabla u\|_{L^{1}\left(\mathbb{R}^{n}\right)}, \quad \forall u \in C_{0}^{\infty}\left(\mathbb{R}^{n}\right)
$$

holds with the same best constant of the isoperimetric inequality (3).

Following this idea, Maz'ya was able to characterize more general inequalities like

$$
\|u\|_{L^{q}(\Omega, \mu)} \leq C\left(\|\nabla u\|_{L^{p}(\Omega)}+\|u\|_{L^{p}(\Omega)}\right), \quad 1 \leq p \leq q
$$

where $\mu$ is a measure and

$$
\|u\|_{L^{q}(\Omega, \mu)}=\left(\int_{\Omega}|u|^{q} d \mu\right)^{1 / q} .
$$

For example, he proved that the inequality

$$
\|u\|_{L^{q}(\Omega, \mu)} \leq C\|\nabla u\|_{L^{1}(\Omega)}, \quad \forall u \in C_{0}^{\infty}(\Omega)
$$

holds if and only if the following isoperimetric inequality is valid

$$
\mu(g)^{1 / q} \leq C \mathcal{H}_{n-1}(\partial g), \quad \forall g \Subset \Omega, \partial g \in C^{\infty}
$$

and the best constants in (4) and (5) coincide. 
Let us consider the $L^{p}$ norm of the gradient $(p>1)$ instead of the $L^{1}$ norm in (4), i.e.

$$
\|u\|_{L^{q}(\Omega, \mu)} \leq D\|\nabla u\|_{L^{p}(\Omega)}, \quad \forall u \in C_{0}^{\infty}(\Omega), \quad q \geq p \geq 1 .
$$

Maz'ya proved that this inequality is equivalent to the "isocapacitary" inequality

$$
\mu(g)^{p / q} \leq C \operatorname{cap}_{p} g, \quad g \Subset \Omega, \partial g \in C^{\infty},
$$

where the $p$-capacity cap $p$ is defined as

$$
\operatorname{cap}_{p} F=\inf \left\{\int_{\Omega}|\nabla \varphi|^{p} d x: \varphi \in C_{0}^{\infty}(\Omega),\left.\varphi\right|_{F} \geq 1\right\}
$$

More precisely, Maz'ya proved that

- if (6) holds, then (7) is true and $D \geq C^{1 / p}$;

- if (7) is valid, then (6) holds and $D \leq p(p-1)^{(1-p) / p} C^{1 / p}$.

This is only the beginning of the story. The equivalence between integral and isoperimetric/isocapacitary inequalities proved to be extremely fruitful. The Maz'ya book "Sobolev Spaces", of which a second enlarged edition has been published in 2010, contains deep developments of this idea, which lead to several applications to the solvability of boundary value problems for elliptic equations and to theorems on the structure of the spectrum of the corresponding operators.

\section{Counterexamples related to the 19th and 20th Hilbert problem}

The celebrated theorem proved indipendently by De Giorgi and Nash in 1957 states that every solution $u \in W^{1,2}$ of the linear elliptic scalar equation

$$
\partial_{i}\left(a_{i j}(x) \partial_{j} u\right)=0
$$

with variable real valued bounded coefficients satisfies Hölder's condition. This result implies the infinite differentiability (or the analyticity) of $u$. This has turned out to be essential for the complete solution of the 19th Hilbert's problem. For many years people tried to extend this result to equations of higher order or to systems. It was a kind of shock when Maz'ya proved that the result does not hold for equations of higher order. Let us describe his counterexample which appeared in the paper Maz'ya, V. Examples of nonregular solutions of quasilinear elliptic equations with analytic coefficients. Funkcional. Anal. i Priložen. 2:3, (1968) 53-57 (Russian). English translation: Functional Anal. Appl. 2 (1968), 230-234.

Let $A$ be the following fourth order differential operator

$$
A u \equiv \nu \Delta^{2}+\kappa \Delta\left(\frac{x_{i} x_{j}}{|x|^{2}} u_{x_{i} x_{j}}\right)+\kappa\left(\frac{x_{i} x_{j}}{|x|^{2}} \Delta u\right)_{x_{i} x_{j}}+\mu\left(\frac{x_{i} x_{j} x_{k} x_{l}}{|x|^{4}} u_{x_{i} x_{j}}\right)_{x_{k} x_{l}}
$$


where the real constants $\nu, \kappa$ and $\mu$ are such that $\nu>0, \kappa^{2}<\mu \nu$. The last condition implies that the operator $A$ is strongly elliptic.

Consider the equation $A u=0$ in $B_{1}=\left\{x \in \mathbb{R}^{n}|| x \mid<1\right\}$. This can be considered as Euler's equation for the functional

$$
\int_{B_{1}}\left[v(\Delta u)^{2}+2 \kappa \frac{x_{i} x_{j}}{|x|^{2}} u_{x_{i} x_{j}} \Delta u+\mu\left(\frac{x_{i} x_{j}}{|x|^{2}} u_{x_{i} x_{j}}\right)^{2}\right] d x \text {. }
$$

The function $u(x)=|x|^{a}$, where

$$
a=2-\frac{n}{2}+\sqrt{\frac{n^{2}}{4}-\frac{(n-1)(\kappa n+\mu)}{v+2 \kappa+\mu}},
$$

belongs to $W^{2,2}$ and is solution of the equation $A u=0$ in $B_{1}$.

If $\kappa=n(n-2), \mu=n^{2}, v=(n-2)^{2}+\varepsilon(\varepsilon>0)$ the strong ellipticity condition is satisfied and the exponent $a$ is equal to

$$
a(\varepsilon)=2-\frac{n}{2}+\frac{n}{2} \sqrt{\frac{\varepsilon}{4(n-1)^{2}+\varepsilon}} .
$$

One can check that, if $n>4$ and $\varepsilon$ is sufficiently small, the solution $|x|^{a(\varepsilon)}$ is not bounded around the origin. This shows that a solution in $W^{2,2}$ of the equation $A u=0$ does not need to be bounded.

In the same paper Maz'ya shows how to construct similar counterexamples for elliptic equations of any order $2 l$ and for quasilinear equations.

This is how Maz'ya described the discovery of this counterexample in his book "Differential Equations of my young years" (p.180): Whatever I did to justify analyticity of solutions of variational problems of higher order, nothing worked. I was stealing up to the problem in various ways, but the solution sneaked off. The problem did not want to be solved for the life of me! But one day, feeling desperate I decided to consider concrete examples in order to understand at least something, and almost at once found that the hypothesis of analyticity was wrong - this was not expected by anyone! Nina Nikolaevna Uraltseva was the first whom I showed my counterexamples. She frowned saying "It's impossible!", but took my manuscript home and promised to check it. A week later she announced for all to hear at the Big Seminar that I was right.

Later Maz'ya with Nazarov and Plamenevskii show that also scalar strongly elliptic second-order differential equations in divergence form with measurable bounded complex coefficients in $\mathbb{R}^{n}(n>4)$ can have generalized solutions which are not bounded in any neighborhood of an interior point of the domain (see Maz'ya, Nazarov, Plamenevskij: Asymptotic Theory of Elliptic Boundary Value Problems in Singularly Perturbed Domains. Vol. I, 2000, pp.391-393).

While for $n=2$ it is known that a generalized solution has to be Hölder continuous (see Morrey (1938), Trans. Am. Math. Soc.), the cases $n=3$ and $n=4$ are still unsolved. 
This problem is contained in a very interesting collection of open problems which Vladimir Maz'ya recently published (Seventy Five (Thousand) Unsolved Problems in Analysis and Partial Differential Equations, Integral Equations and Operator Theory, 2018). They concern function theory, functional analysis, theory of linear and nonlinear partial differential equations.

\section{Multipliers in Sobolev spaces}

Vladimir Maz'ya and Tatyana Shaposhnikova developed a deep theory of multipliers in spaces of differentiable functions. By a multiplier acting from one function space $S_{1}$ into another $S_{2}$, they mean a function which defines a bounded linear mapping of $S_{1}$ into $S_{2}$ by pointwise multiplication. In their theory, the role of the spaces $S_{1}$ and $S_{2}$ is played by Sobolev spaces, Bessel potential spaces, Besov spaces, and the like.

In their book Theory of Sobolev Multipliers (the second very much enlarged edition appeared in 2009) they describe this theory by proving a lot of results, like characterization of multipliers, trace inequalities, relations between spaces of Sobolev multipliers and other function spaces, and so on.

They provide also several applications to analysis, partial differential and integral equations. As they write in the Introduction of the book, they believe that the calculus of Sobolev multipliers provides an adequate language for future work in the theory of linear and nonlinear differential and pseudodifferential equations under minimal restrictions on the coefficients, domains, and other data.

Just to give an example of application of the theory of multiplers, let us consider elliptic boundary value problems in domains with "non-regular" boundaries. Let me introduce some notation. The space of multipliers acting from the Sobolev space $W_{p}^{m}$ into $W_{p}^{l}(1 \leq p<\infty, m \geq l \geq 0)$ is denoted by $M\left(W_{p}^{m} \rightarrow W_{p}^{l}\right)$. Therefore saying that $\gamma \in M\left(W_{p}^{m} \rightarrow W_{p}^{l}\right)$ means that the pointwise moltiplication $u \rightarrow \gamma u$ defines a linear and continuos operator from $W_{p}^{m}$ into $W_{p}^{l}$.

Let $\Omega$ be an open subset of $\mathbb{R}^{n}$ and let $P$ be the operator

$$
P u=\sum_{|\alpha|,|\beta| \leq h}(-1)^{|\alpha|} D^{\alpha}\left(a_{\alpha \beta}(x) D^{\beta} u\right)
$$

where $a_{\alpha \beta} \in C^{l-h}(\bar{\Omega}), l \geq h$. Let us suppose that the Gårding inequality

$$
\operatorname{Re} \int_{\Omega} \sum_{|\alpha|=|\beta|=h} a_{\alpha \beta}(x) D^{\alpha} u \overline{D^{\beta} u} d x \geq C\|u\|_{W_{2}^{h}(\Omega)}^{2}
$$

holds for any $u \in C_{0}^{\infty}(\Omega)$. This implies that the equation $P u=f$ with $f \in W^{-h}(\Omega)$ is uniquely solvable in $\mathscr{W}^{h}(\Omega)$. 
The function $u$ is said to be a solution of the generalized Dirichlet problem if

$$
\left\{\begin{array}{l}
u \in W_{p}^{l}(\Omega) \\
P u=f \text { in } \Omega \\
u-g \in W_{p}^{l}(\Omega) \cap \stackrel{\circ}{W}_{p}^{h}(\Omega),
\end{array}\right.
$$

where $f \in W_{p}^{l-2 h}(\Omega)$ and $g \in W_{p}^{l}(\Omega)$ are given.

Let us suppose that the boundary of $\Omega$ satisfies a condition expressed in terms of multipliers. Specifically, let us suppose that $\partial \Omega \in W_{p}^{l+1-h-1 / p}$ if $p(l-h)>n$ or $\partial \Omega \in M_{p}^{l+1-h-1 / p}(\delta)$ if $p(l-h) \leq n$. The latter means that for each point of the boundary there exists a neighborhood $U$ and a Lipschitz function $\varphi$ such that

$$
U \cap \Omega=\left\{(x, y) \in U \mid x \in \mathbb{R}^{n-1}, y>\varphi(x)\right\}
$$

and

$$
\left\|\nabla \varphi ; \mathbb{R}^{n-1}\right\|_{M W_{p}^{l-1-1 / p}} \leq \delta
$$

Here $\delta$ is a small constant and $M W_{p}^{s}$ is the space of multipliers in $W_{p}^{s}$ for $s>0$ and $L^{\infty}$ for $s \leq 0$.

Under these conditions on the boundary, Maz'ya and Shaposhnikova proved the following existence result:

Given $f \in W_{p}^{l-2 h}(\Omega), g \in W_{p}^{l}(\Omega)$, the BVP (8) has one and only one solution $u \in W_{p}^{l}(\Omega)$.

They show also that a simple sufficient condition which implies their assumption on the boundary when $p(l-h) \leq n$ is

$$
\int_{0}^{1}\left[\omega_{l-h}(t) / t\right]^{p} d t<\infty,
$$

where $\omega_{l-h}$ is the modulus of continuity of the vector-function $\nabla_{l-h} \varphi, \varphi$ being the function in (9).

\section{$5 L^{p}$-dissipativity of partial differential operators}

In a series of joint papers with Vladimir Maz'ya we have considered the problem of characterizing the $L^{p}$-dissipativity of partial differential operators with complex coefficients. I recall that a linear operator $A$ defined on $D(A) \subset L^{p}(\Omega)$ and with range in $L^{p}(\Omega)$ is said to be $L^{p}$-dissipative if

$$
\mathbb{R e} \int_{\Omega}\langle A u, u\rangle|u|^{p-2} d x \leq 0
$$

for any $u \in D(A)$. Here $\Omega$ is a domain in $\mathbb{R}^{n}$ and the functions $u$ are complex valued. 
One of our results gives a necessary and sufficient condition for the $L^{p}$-dissipativity of the partial differential operator with complex valued coefficients

$$
A u=\operatorname{div}(\mathscr{A} \nabla u)
$$

$\left(a^{h k} \in L^{\infty}(\Omega), \Omega \subset \mathbb{R}^{n}, 1<p<\infty\right)$. Specifically, under the assumption that $\operatorname{Im} \mathscr{A}$ is symmetric (i.e. $\operatorname{Im} \mathscr{A}^{t}=\operatorname{Im} \mathscr{A}$ ), we have proved that $A$ is $L^{p}$-dissipative if and only if

$$
|p-2||\langle\operatorname{Im} \mathscr{A}(x) \xi, \xi\rangle| \leq 2 \sqrt{p-1}\langle\mathbb{R e} \mathscr{A}(x) \xi, \xi\rangle
$$

for almost any $x \in \Omega$, for any $\xi \in \mathbb{R}^{n}$.

Examples show that if $\operatorname{Im} \mathscr{A}$ is not symmetric or if there are lower order terms, this result is not true. In general condition (11) is only necessary.

If $I \mathrm{~m} \mathscr{A}$ is symmetric, (11) is equivalent to the condition

$$
\frac{4}{p p^{\prime}}\langle\operatorname{Re} \mathscr{A}(x) \xi, \xi\rangle+\langle\mathbb{R e} \mathscr{A}(x) \eta, \eta\rangle-2(1-2 / p)\langle\operatorname{Im} \mathscr{A}(x) \xi, \eta\rangle \geq 0
$$

for almost any $x \in \Omega$ and for any $\xi, \eta \in \mathbb{R}^{n}$.

More generally, if the matrix $\operatorname{Im} \mathscr{A}$ is not symmetric, the condition

$$
\frac{4}{p p^{\prime}}\langle\operatorname{Re} \mathscr{A}(x) \xi, \xi\rangle+\langle\mathbb{R e} \mathscr{A}(x) \eta, \eta\rangle+2\left\langle\left(p^{-1} \operatorname{Im} \mathscr{A}(x)+p^{\prime-1} \operatorname{Im} \mathscr{A}^{*}(x)\right) \xi, \eta\right\rangle \geq 0
$$

for almost any $x \in \Omega$ and for any $\xi, \eta \in \mathbb{R}^{n}\left(p^{\prime}=p /(p-1)\right)$ is only sufficient for the $L^{p}$-dissipativity of $A$.

Recently several authors have considered the class of operators such that the form (12) is not merely non-negative, but strictly positive, i.e. there exists $\kappa>0$ such that

$$
\begin{aligned}
& \frac{4}{p p^{\prime}}\langle\operatorname{Re} \mathscr{A}(x) \xi, \xi\rangle+\langle\mathbb{R e} \mathscr{A}(x) \eta, \eta\rangle+2\left\langle\left(p^{-1} \mathbb{I m} \mathscr{A}(x)+p^{\prime-1} \mathbb{I m} \mathscr{A}^{*}(x)\right) \xi, \eta\right\rangle \\
& \quad \geq \kappa\left(|\xi|^{2}+|\eta|^{2}\right)
\end{aligned}
$$

for almost any $x \in \Omega$ and for any $\xi, \eta \in \mathbb{R}^{n}$. These operators, which could be called p-strongly elliptic, are playing an increasingly important role in the study of differential operators with complex coefficients, in particular in the study of boundary value problems with $L^{p}$ data. We remark that, if $p=2$, condition (13) reduces to the classical strong ellipticity condition

$$
\operatorname{Re}\langle\mathscr{A}(x) \zeta, \zeta\rangle \geq \kappa|\zeta|^{2}
$$

for almost any $x \in \Omega$ and for any $\zeta \in \mathbb{C}^{n}$.

We have characterized the $L^{p}$-dissipativity also for other classes of operators. Here I want just to mention a characterization of $L^{p}$-dissipativity we have obtained for the the system of linear elasticity

$$
E u=\Delta u+(1-2 v)^{-1} \nabla \operatorname{div} u
$$


( $v$ being the Poisson ratio, $v>1$ or $v<1 / 2$ ). In the planar case we proved that the operator $E$ is $L^{p}$-dissipative if and only if

$$
\left(\frac{1}{2}-\frac{1}{p}\right)^{2} \leq \frac{2(v-1)(2 v-1)}{(3-4 v)^{2}} .
$$

The condition (15) is necessary for the $L^{p}$-dissipativity of operator (14) in any dimension, even when the Poisson ratio is not constant. At the present it is not known if condition (15) is also sufficient for the $L^{p}$-dissipativity of elasticity operator for $n>2$, in particular for $n=3$. This is another open problem contained in the above mentioned collection "Seventy Five (Thousand) Unsolved Problems in Analysis and Partial Differential Equations".

Our results on $L^{p}$-dissipativity can be found in the monograph Cialdea-Maz'ya, Semi-bounded Differential Operators, Contractive Semigroups and Beyond, Operator Theory: Advances and Applications, 243, Birkhäuser, Berlin (2014), where they are considered in the more general frame of semi-bounded operators.

Very recently (Cialdea-Maz'ya, Criterion for the functional dissipativity of second order differential operators with complex coefficients, to appear) we have introduced the more general concept of functional dissipativity of the operator (10) with respect to a certain function $\varphi$. Here $\varphi$ is a positive function defined on $\mathbb{R}^{+}$such that $s \varphi(s)$ is strictly increasing. Let us denote by $\Phi$ the related Young function

$$
\Phi(t)=\int_{0}^{t} s \varphi(s) d s .
$$

If

$$
\mathbb{R e} \int_{\Omega}\langle\mathscr{A} \nabla u, \nabla(\varphi(|u|) u)\rangle d x \geq 0
$$

for any $u \in \stackrel{H}{H}^{1}(\Omega)$ such that $\varphi(|u|) u \in \stackrel{\circ}{H}^{1}(\Omega)$, we say that the operator $A$ is functional dissipative or $L^{\Phi}$-dissipative, in analogy with the terminology used when $\varphi(t)=t^{p-2}$.

We proved that, if $\operatorname{Im} \mathscr{A}^{t}=\operatorname{Im} \mathscr{A}$, the operator $A$ is $L^{\Phi}$-dissipative if and only if

$$
\left|s \varphi^{\prime}(s)\right||\langle\operatorname{Im} \mathscr{A}(x) \xi, \xi\rangle| \leq 2 \sqrt{\varphi(s)[s \varphi(s)]^{\prime}}\langle\mathbb{R e} \mathscr{A}(x) \xi, \xi\rangle
$$

for almost every $x \in \Omega$ and for any $s>0, \xi \in \mathbb{R}^{N}$.

As for $L^{p}$-dissipativity, this condition leads to define a new class of operators, which we shall call $\Phi$-strongly elliptic. They are the second order scalar operators such that

$$
\begin{aligned}
& {\left[1-\Lambda^{2}(t)\right]\langle\mathbb{R e} \mathscr{A}(x) \xi, \xi\rangle+\langle\mathbb{R e} \mathscr{A}(x) \eta, \eta\rangle} \\
& \quad+[1+\Lambda(t)]\langle\operatorname{Im} \mathscr{A}(x) \xi, \eta\rangle+[1-\Lambda(t)]\left\langle\operatorname{Im} \mathscr{A}^{*}(x) \xi, \eta\right\rangle \geq \kappa\left(|\xi|^{2}+|\eta|^{2}\right)
\end{aligned}
$$


for a certain $\kappa>0$ and for almost every $x \in \Omega$ and for any $t>0, \xi, \eta \in \mathbb{R}^{N}$. Here $\Lambda$ is the function defined by the relation

$$
\Lambda(s \sqrt{\varphi(s)})=-\frac{s \varphi^{\prime}(s)}{s \varphi^{\prime}(s)+2 \varphi(s)} .
$$

\section{History of mathematics}

In 1998 Vladimir Maz'ya and Tatyana Shaposhnikova published the huge volume "Jacques Hadamard. A Universal Mathematician". It contains more than 500 pages and it was later translated in French and in Russian. It is both a biography and a description of Hadamard's mathematics. The book is also full of photographs and pictures which make the reading extremely pleasant.

The authors made an amazing job finding their sources. As Roger Cooke wrote in "Maz'ya's work on the biography of Hadamard" (in Rossmann et al. (eds.), The Maz'ya anniversary collection (Birkhäuser Verlag, Basel, 1999)) although much of the relevant material unfortunately disappeared during World War II, they were able to find a great deal of new material nevertheless. They located Hadamard's grandson, Francis Picard and were able to look at the materials collected by Hadamard's daughter Jacqueline, including her autobiography. They interviewed several mathematicians who had known Hadamard personally, and they submitted their drafts of the new and revised material to experts in a number of areas of mathematics and the history of mathematics, and the history of French mathematics in particular. The result, published in English this time, was a triumph of mathematical biography that one can only hope will be repeated for many other great twentieth-century mathematicians. ...It will probably turn out to be the definitive biography of Hadamard.

Another book written by Maz'ya and recently translated in russian, which gives an important contribution to the history of mathematics is "Differential Equations of My Young Years". It is an autobiography which covers events from 1937 till 1968. The book is full of wonderful photographs. Reading this book means taking a dip in the past. It gives a precise description of the Soviet life in 40s-60s of the last century. Even if the book is full of mathematics, it can be read by anyone. As Havin writes in the foreword, the fact that mathematics appears on many of its pages in no way diminishes the book's clarity of discourse and attraction to a variety of readers.

In addition to more personal events from adolescence and early adulthood, we can read very interesting facts about the russian mathematics in that period.

\section{Other books}

Although Maz'ya's books appearing in Fig. 2 do not concern his scientific activity, I think that they deserve a mention, because they are another proof of the great imagination he has. 

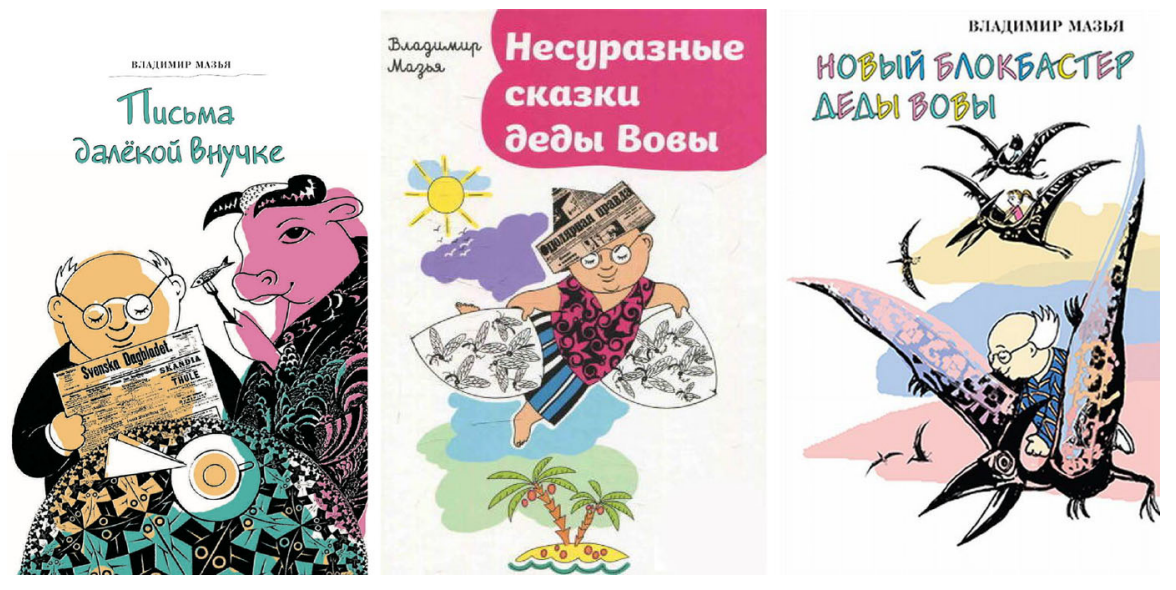

Fig. 2 Maz'ya's fairy tales

They are three books of fairy tales intended for middle school children. The heroes of these stories are animals and people, as well as space aliens and underground inhabitants.

As Gohberg wrote in "Vladimir Maz'ya: friend and mathematician. Recollections." (in Rossmann et al. (eds.), The Maz'ya anniversary collection (Birkhäuser Verlag, Basel, 1999)): whatever he writes is beautiful, his love for art, music and literature seeming to feed his mathematical aesthetic feeling.

Funding Open access funding provided by Università degli Studi della Basilicata within the CRUI-CARE Agreement.

\section{Compliance with ethical standards}

Conflict of interest The author declares that there is no conflict of interest.

Availability of data and materials Not applicable. The manuscript has no associated data.

Open Access This article is licensed under a Creative Commons Attribution 4.0 International License, which permits use, sharing, adaptation, distribution and reproduction in any medium or format, as long as you give appropriate credit to the original author(s) and the source, provide a link to the Creative Commons licence, and indicate if changes were made. The images or other third party material in this article are included in the article's Creative Commons licence, unless indicated otherwise in a credit line to the material. If material is not included in the article's Creative Commons licence and your intended use is not permitted by statutory regulation or exceeds the permitted use, you will need to obtain permission directly from the copyright holder. To view a copy of this licence, visit http://creativecommons.org/licenses/by/4.0/.

Publisher's Note Springer Nature remains neutral with regard to jurisdictional claims in published maps and institutional affiliations. 